American Journal of Applied Sciences 9 (8): 1182-1187, 2012

ISSN 1546-9239

(C) 2012 Science Publications

\title{
Moringa oleifera Leaves Extract Attenuates Neuropathic Pain Induced by Chronic Constriction Injury
}

\author{
${ }^{1,3}$ Jurairat Khongrum, ${ }^{2,3,4}$ Jintanaporn Wattanathorn, \\ ${ }^{2,3,4}$ Supaporn Muchimapura ${ }^{2,3}$ Wipawee Thukhum-mee, \\ ${ }^{3}$ Cholathip Thipkaew, ${ }^{2,3}$ Panakaporn Wannanon and ${ }^{2,3}$ Terdthai Tong-un \\ ${ }^{1}$ Department of Physiology and Graduate School (Neuroscience Program), \\ ${ }^{2}$ Department of Physiology, \\ Faculty of Medicine, Khon Kaen University, Khon Kaen 40002, Thailand \\ ${ }^{3}$ Integrative Complementary Alternative Medicine Research and Development Group, \\ Department of Physiology, Faculty of Medicine, Khon Kaen University, Khon Kaen, Thailand \\ ${ }^{4}$ Center for Research and Development of Herbal Health Product, \\ Faculty of Pharmaceutical, Khon Kaen University, Khon Kaen, Thailand
}

\begin{abstract}
Problem statement: Neuropathic pain, a challenge of this decade, has been reported to be associated with the diversity conditions including diabetes. At present, there are no conventional analgesics that can effectively treat neuropathic pain with a satisfactory outcome. Due to the limitation of therapeutic efficacy, the searching for novel effective remedies in the management of neuropathic pain is required. Approach: Male Wistar rats, weighing 180-220 g were induced diabetes mellitus by Streptozotocin (STZ) (single injection, $65 \mathrm{mg} \mathrm{kg}^{-1} \mathrm{BW}$, i.p). Diabetic rats were induced neuropathic pain by Constricting the right sciatic nerve (CCI) at permanently. Then, all rats were administered the extract of M. oleifera leaves at doses of 100,200 and $300 \mathrm{mg} \mathrm{kg}^{-1} \mathrm{BW}$ once daily in a period of 21 days. The analgesic effect of the plant extract was evaluated using Von Frey filament and hot plate tests every 3 days after CCI throughout 21-day experimental period. In addition, at the end of the experiment, the alteration of oxidative damage markers including MDA level and the activities of SOD, CAT and GSHPX in the injured sciatic nerve were also evaluated. Results: The current results showed that rats subjected to M.oleifera leaves extract at doses of 100 and $200 \mathrm{mg} \mathrm{kg}^{-1} \mathrm{BW}$ significantly reversed the decreased withdrawal threshold intensity and withdrawal latency in Von Frey filament and hot plate tests respectively. In addition, rats subjected to the medium dose extract also reversed the decreased activities of SOD and GSH-Px and the elevation of MDA level in the injured nerve. Taken all together, our data suggest that $M$. oleifera leaves extract can attenuate neuropathic pain in diabetic condition. The possible underlying mechanism may occur partly via the decreased oxidative stress. However, other mechanisms may also involve. Conclusion: Our results suggest that $M$. oleifera leaves may be the potential novel adjuvant therapy for neuropathic pain management.
\end{abstract}

Key words: Thiobarbituric Acid Reactive Substances (TBARS), Streptozotocin (STZ), Dipheny-2Picrylhydrazyl (DPPH), neuropathic pain management, mildly noxious stimulus

\section{INTRODUCTION}

Neuropathic pain has been defined as "pain initiated or caused by a primary lesion or dysfunction in the nervous system" (Campbell and Meyer, 2006). Although, the precise records of neuropathic pain sufferers are not available, it is estimated that more than million people worldwide are suffering from this condition (Hall et al., 2006). This condition is reported to be associated with the diversity conditions including diabetes. Despite its heterogeneous etiology, it is uniformly characterized by stimulus-independent persistent pain or abnormal sensory perception of pain, such as allodynia (a painful response to a normally innocuous stimulus) and hyperalgesia (exaggerated pain sensations as a result of exposure to a mildly noxious stimulus) (Ueda and Rashid, 2003). At present, conventional analgesics that are available in the market

Corresponding Author: Jintanaporn Wattanathorn, Department of Physiology, Faculty of Medicine and The Integrative Complementary Alternative Medicine Research Group, Khon Kaen University, Khon Kaen 40002, Thailand 
cannot exert sufficient effects on the neuropathic pain associated with diabetes and no potentially effective drug for treatment of this type of pain is currently available.

Several lines of evidence have demonstrated that oxidative stress and Cyclo-Oxygenase type 2 (COX-2) contribute the important role in the pathophysiology of neuropathic pain (Khalil et al., 1999; Ma and Quirion, 2008). Based on the limitation of therapeutic efficacy and pathophysiology of neuropathic pain mentioned earlier, the developing novel agents for the management of diabetic neuropathy, including the therapeutic agents derived from natural medicinal plants possessing antioxidant and anti-inflammatory activities, have become an active area of intense research.

Moringa oleifera Lam. or "Marum" in Thailand is belonging to Moringaceae family. It is long-term used as a vegetable or as a food ingredient. It is also used in traditional folklore for treating many ailments such as asthma, spasm, enlarged liver and spleen, infection and nervous debility, ulcer, inflammation and for wound healing (Mishra et al., 2011; Promkum et al., 2010). Recent studies also shows that the extract of M. oleifera leaves also possesses antidiabetic (Jaiswal et al., 2009) and antioxidant (Pari et al., 2007) activities. Due to the antidiabetic and antioxidant effects of M. oleifera, we hypothesized that the leaf extract of this medicinal plant might improve neuropathic pain induced by diabetic condition. To the best of our knowledge, no scientific data is available. Therefore, this study aimed to determine the analgesic effect and possible underlying mechanism of $M$. oleifera leaves extract in animal models of neuropathic pain induced by diabetes mellitus.

\section{MATERIALS AND METHODS}

Plant material: Fresh leaves of M. oleifera were collected during November-December, 2010 from the Khon Kaen province, Thailand.

Plant material preparation: The fresh leaves were immediately cleaned, then cut into small pieces and dried at the temperature less than $50^{\circ} \mathrm{C}$. The dried plant material was ground into fine coarse powder and extracted with $50 \%$ alcohol. After that evaporation of solvent in rotary evaporator affords a crude extract of the soluble components and filtrate was lyophilized using lyophilizer (Flexi-Dry MPTM). The percent yield of the extract was $17.49 \%$. The extracts were stored at $-25^{\circ} \mathrm{C}$ in a dark bottle until used. The crude extract was suspended in $1 \% \mathrm{CMC}$ (Sodium carboxymethylcellulose). The antioxidant activities of the plant extracts were measured by Ferric Reducing Antioxidant Power (FRAP) activity was $114.55 \pm 0.003 \mathrm{mM}$ Ascorbic acid/g extract and the ability to scavenge 1, 1-Dipheny-2Picrylhydrazyl (DPPH) with EC50 values was $274.83 \pm 0.322 \mu \mathrm{g} \mathrm{mL}^{-1}$ (Thaipong et al., 2006).
Animals: The animals were approved by the Animal Ethic Committee of Khon Kaen University. Adult male Wistar rats were used as experimental animals. They were obtained from National Animal Center, Salaya. The weights of the animals on the first day of the experiment were $180-220 \mathrm{~g}$. All animals were randomly housed 5 per cage and maintained at 12-h light-dark cycle and free access to food and water available ad libitum.

Experimental protocol: The rats were divided into Naïve intact groups and diabetic mellitus group. The diabetic mellitus group was subdivided into; Vehicle plus nerve constriction, Vehicle plus sham operation, Moringa oleifera Lam. $100 \mathrm{mg} \mathrm{kg}{ }^{-1} \mathrm{BW}$ plus sciatic nerve constriction, Moringa oleifera Lam. $200 \mathrm{mg}$ $\mathrm{kg}^{-1}$ BW plus sciatic nerve constriction, Moringa oleifera Lam. $300 \mathrm{mg} \mathrm{kg}^{-1} \mathrm{BW}$ plus sciatic nerve constriction and Vitamin C $100 \mathrm{mg} \mathrm{kg}^{-1}$ BW plus sciatic nerve constriction. The animals in diabetic mellitus groups were treated with the assigned substance for a period of 21 days. Then, the nociceptive threshold, which determined the sensory function, was assessed by Von Frey filament and hot plate tests. Whereas the motor function was assessed by the De Medinacelli method after a single dose and every 3 days, until the end of the experimental period.

Induction of neuropathic pain: Diabetes was induced in male Wistar rats by intraperitoneal injection of streptozotocin. Streptozotocin (STZ) was obtained from Sigma Chemicals Co., St. Louis, MO, USA. (STZ; single dose of $65 \mathrm{mg} \mathrm{kg}^{-1}$ ) dissolved in cold sterile $0.9 \%$ saline solution. After $72 \mathrm{~h}$, rats with marked hyperglycemia (fasting blood glucose $\geq 300 \mathrm{mg} \mathrm{dL}^{-1}$ ) were selected and used for further study. The selected rats were anesthetized by ethyl ether. Then, the right sciatic nerve of each rat was exposed at high thigh level. The dorsum of nerve was carefully freed from the surrounding connective tissue at a site just distal to the point at which the posterior biceps semitendinous nerve branches of the common sciatic nerve. The tight ligation around sciatic nerve was performed using chromic gut (4-0 silk). Sham operated rats were exposed to similar surgical conditions without nerve ligation.

Evaluation of sensory function using von Frey filament test: In order to measure sensory function of sciatic nerve foot withdrawal threshold in the response to mechanical stimuli via von Frey filament test was carried out 10 von Frey filaments, with approximately equal logarithmic incremental bending forces were chosen (Wattanathorn et al., 2012). The filaments were applied to the medial plantar surface of each hind paw 
in a series of ascending forces. Each filament was tested five times per paw and the mechanical threshold was defined as the minimal force that caused at least three withdrawals observed out of five consecutive trials (Yalcin et al., 2009).

Determination of foot withdrawal reflex time via hot plate test: In the hot plate test, the withdrawal response latency was measured by stopwatch to a maximum cutoff time of $12 \mathrm{~s}$ at 56 degrees Celsius and $15 \mathrm{~s}$ at 52 degrees Celsius. After the cutoff time, if the rat hind paw still remained on the hot plate, the hind paw was removed from it to prevent heat injury (i.e., blistering). At each time point, the Withdrawal Reflex Latency (WRL) was measured in triplicate and the mean of these three measurements was considered to be the WRL for that time point.

Determination of the superoxide dismutase (SOD), Glutathione Peroxidase (GPx) and Catalase Activities (CAT) and the Malondialdehyde (MDA) level: The rats were divided into various groups as previously described in the experimental protocol. After the last dose of administration, all rats were sacrificed. The sciatic nerve of the lesion side was isolated and prepared as homogeneous for the determination of MDA level and the activities of SOD, CAT and GPx. MDA was estimated by determining the accumulation of Thiobarbituric Acid Reactive Substances (TBARS) (Wattanathorn et al., 2012) in the nerve homogenate whereas the activities of Superoxide Dismutase (SOD), Catalase (CAT) and Glutathione Peroxidase (GSH-Px) were determined by recording the ability to inhibit cytochrome $\mathrm{C}$, the rate of decrease in $\mathrm{H}_{2} \mathrm{O}_{2}$ and the amount of reduced Nicotinamide Adenine Dinucleotide Phosphate (NADPH) oxidized per minute respectively (Jittiwat et al., 2009).

Statistical analysis: Data are expressed as means \pm S.E.M. and were analyzed statistically by one-way ANOVA, followed by Post-hoc (LSD) test. The results were considered statistically significant at $\mathrm{p}<0.05$.

\section{RESULTS}

The effect of the M. oleifera on hyperalgesia: Figure 1 showed that vehicle plus sham operation produced no significant change on withdrawal latency. Rats exposed to vehicle plus Chronic Constriction (CCI) revealed the significant decrease in withdrawal latency since the 6th day after CCI throughout the experimental period ( $<<0.001$ all; compared to vehicle+sham operation). Vitamin C, the antioxidant which previously reported to ameliorate thermal hyperalgesia (Sharma et al., 2009).
Table 1: The effect of $M$. oleifera leaves extract on the oxidative damage maker and the scavenging enzymes in sciatic nerve. Rats were treated with vehicle or M. oleifera at doses of 100,200 and $300 \mathrm{mg} \cdot \mathrm{kg}^{-1} \mathrm{BW}$ once daily at a period of 21 days. After the last treatment, the rats were sacrificed and collected the sciatic nerve. The data were shown as mean \pm S. E. M. ( $n=6$ per group). $* * * * p<0.05$ and 0.001 respectively; compared to vehicle + CCI. ${ }^{\text {aa,aaa }} \mathrm{p}<0.01$ and 0.001 respectively; compared to vehicle+ sham operation

\begin{tabular}{|c|c|c|c|c|}
\hline $\begin{array}{l}\text { Group/ Oxidative } \\
\text { stress maker }\end{array}$ & $\begin{array}{l}\mathrm{MDA} \\
(\mathrm{nmol} / \mathrm{mL})\end{array}$ & $\begin{array}{l}\text { SOD } \\
\text { (U/mg.protein) }\end{array}$ & $\begin{array}{l}\text { GSH-Px } \\
\text { (U/mg.protein) }\end{array}$ & $\begin{array}{l}\text { CAT } \\
\text { (U/mg.protein) }\end{array}$ \\
\hline Control & $0.10 \pm 0.01$ & $3.31 \pm 0.31$ & $3.75 \pm 0.12$ & $2.56 \pm 0.10$ \\
\hline $\begin{array}{l}\text { Vehicle }+ \\
\text { sham operation }\end{array}$ & $0.14 \pm 0.02$ & $3.70 \pm 0.76$ & $3.71 \pm 0.32$ & $2.965 \pm 0.21$ \\
\hline Vehicle + CCI & $0.92 \pm 0.11^{\text {aaa }}$ & $1.01 \pm 0.23^{\mathrm{aa}}$ & $1.75 \pm 0.03^{\text {aa }}$ & $1.23 \pm 0.03^{\mathrm{aa}}$ \\
\hline $\begin{array}{l}\text { Vitamin C } 100 \\
\mathrm{mg} \mathrm{kg}^{-1} \mathrm{BW}\end{array}$ & $0.33 \pm 0.01 * * *$ & $2.18 \pm 0.14$ & $2.75 \pm 0.16$ & $2.18 \pm 0.10$ \\
\hline $\begin{array}{l}\text { M.olifera } 100 \\
\mathrm{mg} \mathrm{kg}^{-1} \mathrm{BW}\end{array}$ & $0.39 \pm 0.05 * * *$ & $2.01 \pm 0.21$ & $1.93 \pm 0.09$ & $1.74 \pm 0.10$ \\
\hline $\begin{array}{l}\text { M.olifera } 200 \\
\mathrm{mg} \mathrm{kg}^{-1} \mathrm{BW}\end{array}$ & $0.21 \pm 0.03 * * *$ & $3.31 \pm 0.33^{*}$ & $2.938 \pm 0.12 *$ & $2.21 \pm 0.07$ \\
\hline $\begin{array}{l}\text { M.olifera } 300 \\
\mathrm{mg} \mathrm{kg}^{-1} \mathrm{BW}\end{array}$ & $0.71 \pm 0.03$ & $1.92 \pm 0.51$ & $1.94 \pm 0.13$ & $1.51 \pm 0.09$ \\
\hline
\end{tabular}

It was found that rats subjected to $M$. oleifera treatment at a dose of $200 \mathrm{mg} \mathrm{kg}^{-1} \mathrm{BW}$ and vitamin C significantly attenuated the decreased withdrawal latency induced by CCI since 12nd day after CCI and these significant changes were observed throughout the study period ( $\mathrm{p}<0.05, .05, .05$ and .01 respectively both vitamin $\mathrm{C}$ and $M$. oleifera at a dose of $200 \mathrm{mg}$. $\mathrm{kg}^{-1} \mathrm{BW}$; compared to vehicle plus $\mathrm{CCI}$ ). When the treatment duration was prolonged further to 18 th and 21 st day after $\mathrm{CCI}$, rats which received $M$. oleifera extract at a dose of $100 \mathrm{mg}$. $\mathrm{kg}^{-1} \mathrm{BW}$ also significantly mitigated the decreased withdrawal latency $(\mathrm{p}<0.05$ all; compared to vehicle plus $\mathrm{CCI}$ ).

In addition to heat hyperalgesia, the effect of M.oleifera leaves extract on mechanical hyperalgesia was also determined. The results were shown in Fig. 2. The rats which subjected to vehicle plus CCI showed the significant reduction of withdrawal latency when they were stimulated by mechanical stimuli via Von Frey filament since the 3rd day after CCI $(p<0.01$; compared to vehicle plus sham operation). This significant change was still observed when the treatment duration was increased further to $6,9,12,15$, 18, 21 days after CCI $(\mathrm{p}<0.01, .001, .05, .01$ and .01 respectively; compared to vehicle plus CCI). Rats exposed to vitamin $\mathrm{C}$ treatment and M.oleifera extract at a dose of $200 \mathrm{mh} \cdot \mathrm{kg}^{-1} \mathrm{BW}$ plus CCI significantly attenuated the decreased withdrawal latency induced by CCI ( $p<0.05$ all; compared to vehicle plus CCI).

Effect of M.oleifera on oxidative stress damage markers: The effects of M.oleifera on oxidative stress markers comprising of MDA level ant the activities of SOD, CAT and GSH-Px were also assessed and data were shown in Table 1. 


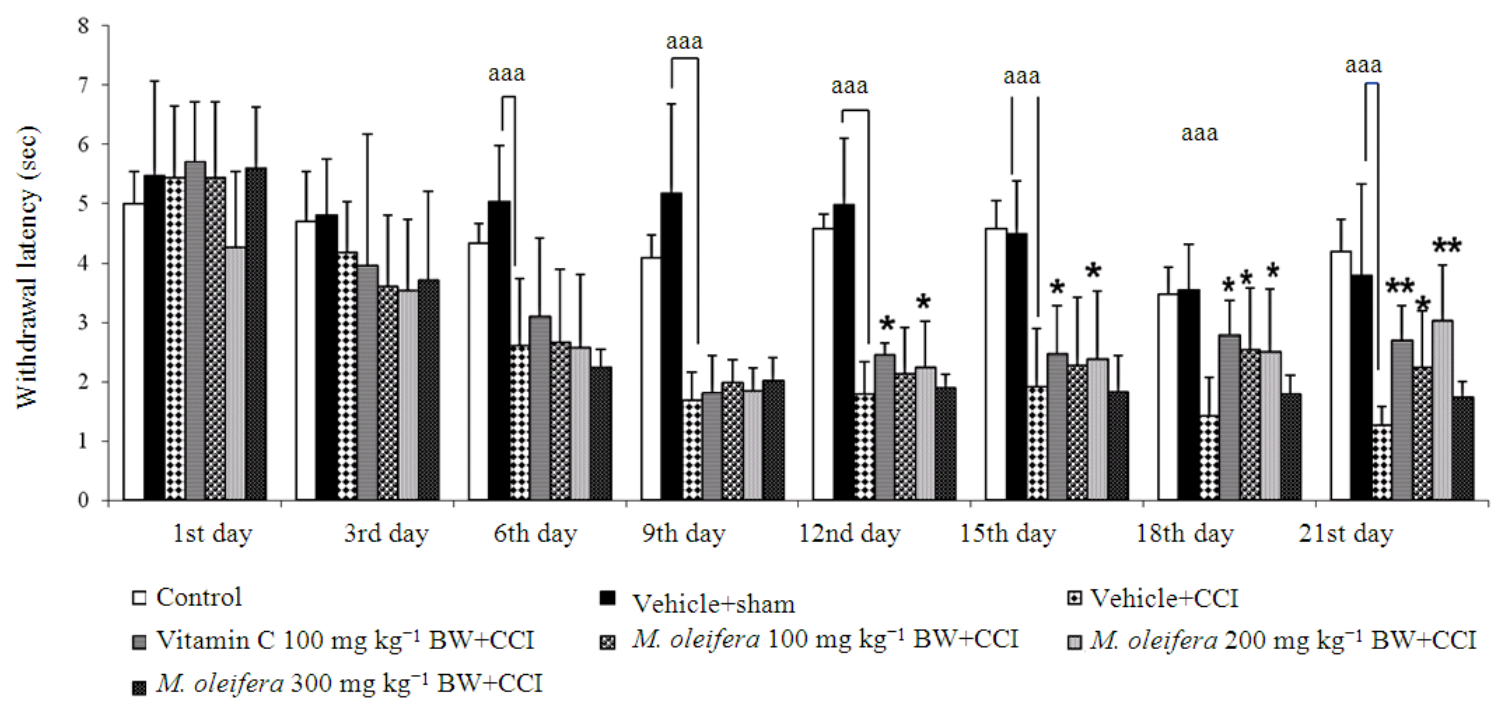

Fig. 1: The effect of M.oleifera leaves extract on heat hyperalgesia. Rats were treated with vehicle or M.oleifera at doses of 100, 200 and $300 \mathrm{mg} \mathrm{kg}^{-1} \mathrm{BW}$ once daily for a period of 21 days. The data were shown as mean \pm S.E.M. ( $\mathrm{n}=6$ per group). ${ }^{*}, * * \mathrm{p}<0.05$ and 0.01 respectively; compared to vehicle + CCI. ${ }^{\text {aaa }}$ p-value $<0.001$ compared to vehicle + sham operation

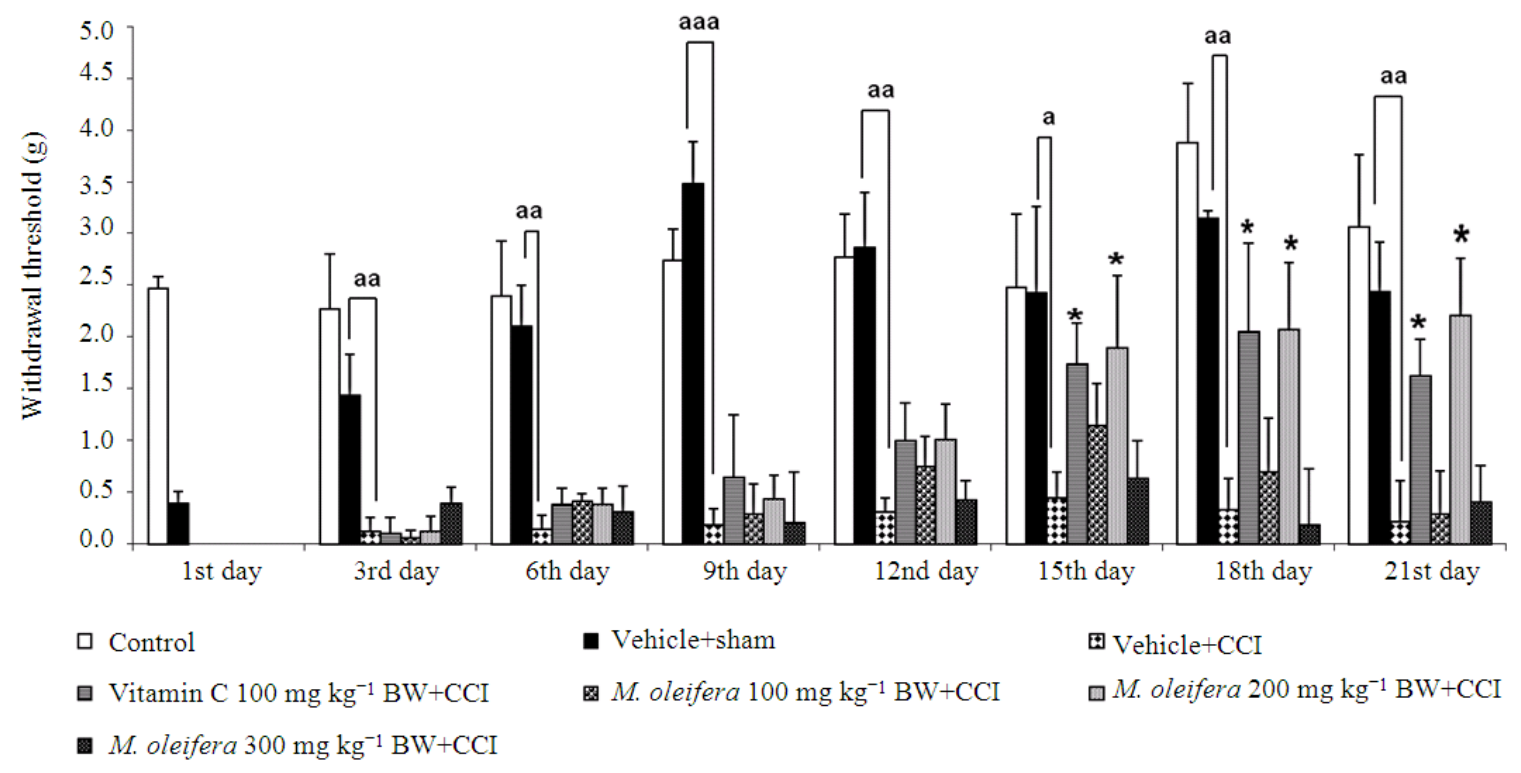

Fig. 2: The effect of M.oleifera leaves extract on mechanical hyperalgesia. Rats were treated with vehicle or $M$. oleifera at doses of 100, 200 and $300 \mathrm{mg}$. $\mathrm{kg}^{-1} \mathrm{BW}$ once daily for a period of 21 days. The data were shown as mean \pm S.E.M. ( $\mathrm{n}=6$ per group). ${ }^{*} \mathrm{p}<0.05$ compared to vehicle + CCI. ${ }^{\text {a,aa,aaa }} \mathrm{p}<0.05,0.01$ and 0.001 respectively; compared to vehicle+ sham operation

Both sham operation and vehicle treatment produced no significant change on all parameters mentioned earlier. Rats subjected to vehicle plus CCI treatment significantly decreased the activities of SOD, GSH-Px and CAT ( $\mathrm{p}<0.001,0.01,0.01$ and 0.01 respectively; compared to vehicle+sham operation). Both vitamin C and low dose of the extract significantly elevated the enhanced MDA level without the significant changes of scavenger enzymes. However, rats subjected to M.oleifera extract at a dose of $200 \mathrm{mg} \cdot \mathrm{kg}^{-1} \mathrm{BW}$ 
significantly reversed the reduction of SOD and GSHPx activity and the decrease of MDA level $(\mathrm{p}<0.01, .01$ and 0.001 respectively; compared to vehicle+CCI).

\section{DISCUSSION}

To date, the most reliable, quantitative and reproducible method for the sensation recovery of nerve in neuropathic pain are anti-nociceptive tests via Von Frey filament test and hot plate test. The significant reduction in withdrawal threshold and withdrawal latency (allodynia threshold) observed at 9 days after CCI confirms the hyperalgesic effect.

Previous studies had revealed that streptozotocininduced diabetes is accompanied by chronic oxidative stress due to the hyperglycemia (Low et al., 1997). Hyperglycemia could enhance the generation of reactive oxygen species which in turn gave rise to the increased neuronal damage by oxidizing proteins, lipids and augmented levels of lipid peroxidation products in cellular membranes (Hawkins and Davies, 2001; Baydas et al., 2005). Oxidative damage occurring in the peripheral neurons and peripheral nerves induced by oxidative stress often lead to an augmented sensitivity to painful stimuli and peripheral neuropathic pain (Serpell, 2005). Several lines of evidence have indicated that various ROS scavengers could reduce pain behaviors predominantly through peripheral nerve protection (Dam et al., 1998; Halat and Dennehy, 2003).

In the present study, we have demonstrated that Moringa oleifera leaves extract could attenuate hyperalgesia and the elevation of oxidative stress induced by sciatic nerve constriction injury in diabetic rats. Our results clearly revealed that M.oleifera leaves extract at a dose of $200 \mathrm{mg} \mathrm{kg}^{-1}$ BW could enhance SOD and GSHPx activities but decreased MDA level in accompany with the attenuation of hyperalgesia in the injured nerve. However, the low dose of this plant extract could attenuate the hyperalgesia condition in accompany with the decreased MDA level while no significant changes of the activities of SOD, CAT and GSH-PX were observed. Therefore, the decreased MDA level in rats subjected to M.oleifera leaves extract at low dose might be due to either the decreased oxidative stress formation or due to the enhanced non-enzymatic scavenging activity. In addition, the possible underlying mechanism contributing the important role on the analgesic effect of $M$. oleifera leaves extract may attribute not only to the decreased oxidative stress damage but also to other mechanisms. Based on the previous finding that both the inhibition of calcium channel and Cyclo-Oxygenase 2 (COX-2) are also playing the role on the hyperalgesia and allodynia in neuropathic pain condition (Muthuraman and Singh,
2011), we do suggest that the mechanism just mentioned may also contribute the role in the analgesic effect of $M$. oleifera extract especially at a low dose concentration (100 $\mathrm{mg} \mathrm{kg}^{-1} \mathrm{BW}$ ).

\section{CONCLUSION}

The possible mechanism underlying these results may be $M$. oleifera exert the antioxidant effect. However, these, the precise underlying mechanism still require further investigation.

\section{ACKNOWLEDGEMENT}

"This study was supported by the Higher Education Research Promotion and National Research University Project of Thailand, Office of the Higher Education Commission, through the Food and Functional Food Research Cluster of Khon Kaen University" and Center for Research and Development of Herbal Health Product.

\section{REFERENCES}

Baydas, G., E. Sonkaya, M. Tuzcu, A. Yasar and E. Donder, 2005. Novel role for gabapentin in neuroprotection of central nervous system in streptozotocine-induced diabetic rats. Acta Pharmacol. Sin., 26: 417-422. DOI: 10.1111/j.1745-7254.2005.00072.x

Campbell, J.N. and R.A. Meyer, 2006; Mechanisms of Neuropathic Pain. Neuron., 52: 77-92. Doi: 10.1016/j. neuron. 2006.09.021

Dam, P.S.V., B.S. Van Asbeck, B. Bravenboer, J.F. Van Oirschot and W.H. Gispen et al., 1998. Nerve function and oxidative stress in diabetic and vitamin E-deficient rats. Free Radic. Biol. Med., 24: 18-26. DOI: 10.1016/S0891-5849(97)00122-6

Halat, K.M. and C.E. Dennehy, 2003. Botanicals and dietary supplements in diabetic peripheral neuropathy. J. Am. Board Fam Pract., 16: 47-57. PMID: 12583650

Hall, G., D. Carroll, D. Parry and H.J. McQuay, 2006. Epidemiology and treatment of neuropathic pain: The UK primary care perspective. Pain, 122: 156162. PMID: 16545908

Hawkins, C.L. and M.J. Davies, 2001. Generation and propagation of radical reactions of proteins. Biochem Biophys Acta., 1504: 196-219. PMID: 11245785

Jaiswal, D., P. Kumar Rai, A. Kumar, S. Mehta, G. Watal, 2009. Effect of Moringa oleifera Lam. Leaves aqueous extract therapy in hyperglycemic rats. J Ethnopharmacolo., 123: 392-396. PMID: 19501271 
Jittiwat, J., J. Wattanthorn, S. Muchimapura and C. Bunchonglikikul, 2009. Porcine brain extract attenuates memory impairments induced by focal cerebral ischemia. Am. J. Applied Sci., 6: 16621668. DOI: 10.3844/ajassp. 2009.1662.1668

Khalil, Z., T. Liu and R.D. Helme, 1999. Free radicals contribute to the reduction in peripheral vascular responses and the maintenance of thermal hyperalgesia in rats with chronic constriction injury. Pain, 79: 31-37. PMID: 9928773

Low, P.A., K.K. Nickander and H.J. Tritschler, 1997. The roles of oxidative stress and antioxidant treatment in experimental diabetic neuropathy. Diabetes, 46: S38-S42. PMID: 9285497

Ma, W. and R. Quirion, 2008. Does COX2-dependent PGE2 play a role in neuropathic pain. Neurosci. Lett., 437: 165-169. PMID: 18434017

Mishra, G., P. Singh, R. Verma, S. Kumar and S. Srivastav et al., 2011. Traditional uses, Phytochemistry and pharmacological properties of Moringa oleifera plant: An overview. Der Phamacia Lett., 3: 141-164.

Muthuraman, A. and N. Singh, 2011. Attenuating effect of Acorus Calamus extract in the chronic constriction injury induced neuropathic pain in rats: An evidence of anti-oxidative, antiinflammatory, neuroprotective and calcium inhibitory effects. BMC Complement Altern Med., 11: $1-14$.

Promkum, C., P. Kupradinun, S. Tuntipopipat, C. Butryee, 2010. Nutritive evaluation and effect of Moringa oleifera pod on clastogenic potential in the mouse. Asian Pac. J. Cancer Prev., 11: 627632. PMID: 19966143

Pari, L., M. Karamac, A. Kosinska, A. Rybarczyk and R. Amarowicz, 2007. Antioxidant activity of the crude extracts of drumstick tree (Moringa oleifera Lam.) and sweet brommweed (Soparia ducist L) leaves. Polish J. Food Nutrition Sci., 57: 201-208.
Serpell, M., 2005. Anatomy, physiology and pharmacology of pain. Anaesth Intensive Care Med., 6: 7-10. DOI: 10.1383/anes.6.1.7.57133

Sharma, M., T. Katyal, G. Grewal, D. Behera and R.D. Budhiraja, 2009. Effect of antioxidants such as $\beta$ carotene, vitamin $\mathrm{C}$ and vitamin $\mathrm{E}$ on oxidative stress, thermal hyperalgesia and cold allodynia in streptozotocin induced diabetic rats. The Internet J. Pharmacol.

Thaipong, K., L. Cisneros-Zevallosc, D. H. Byrnec, 2006. Comparison of ABTS, DPPH, FRAP and ORAC assays for estimating antioxidant activity of guava fruit extracts. J. Food Composition Anal., 19: 669-675. DOI: 10.1016/j. jfca. 2006.01.003

Ueda, H. and H. Rashid, 2003. Molecular mechanism of neuropathic pain. Drug News Perspect, 16: 605613. PMID: 14702142

Wattanathorn, J., N. Sattroopinat, T. Tong-Un, S. Muchmapura and P. Wannanond et al., 2012. Neuroprotective effect against cerebral ischemia of Passiflora foetida. Am. J. Applied Sci., 9: 600-604. DOI: 10.3844/ajassp. 2012.600.604

Yalcin, I. and N. Choucair-Jaafar, M. Benbouzid, L.H. Tessier and A. Muller et al., 2009. Beta2adrenoceptors are critical for antidepressant treatment of neuropathic pain. Ann. Neurol., 65: 218-225. PMID: 19259968 